\title{
Primates and Dung Beetles: Two Dispersers Are Better than One in Secondary Forest
}

\author{
Laurence Culot ${ }^{1,2,3}$ (D) Marie-Claude Huynen ${ }^{2}$. \\ Eckhard W. Heymann ${ }^{3}$
}

Received: 28 September 2017 / Accepted: 22 March 2018 / Published online: 17 May 2018

(C) Springer Science+Business Media, LLC, part of Springer Nature 2018

\begin{abstract}
Primary seed dispersal by primates (phase I) followed by secondary seed dispersal by dung beetles (phase II) is a common diplochorous system in tropical forests. In such systems, phase I affects the occurrence/outcome of phase II, triggering cascading effects along the chain of plant recruitment with direct consequences on seed dispersal effectiveness. However, we know very little regarding whether seed dispersal effectiveness is increased or decreased by phase II and whether this effect is consistent among habitats. Using a primate-dung beetle diplochorous system, we determined 1) the characteristics of phase I that may affect phase II; 2) the pathways relating biotic/ abiotic factors to seed/seedling survival; and 3) if the direction and/or magnitude of phase II effects on seed dispersal effectiveness depend on phase I characteristics. We marked and characterized the dispersal characteristics of 981 seeds dispersed by two tamarin species (Saguinus mystax, Leontocebus nigrifrons) and checked the fate of 503 of them for $\geq 1$ year. Seeds dispersed by L. nigrifrons and seeds surrounded by larger amounts of dung were more likely to be buried by dung beetles. Burial increased seed survival in secondary forest while low seed density increased germination in both habitats. Seed burial increased seed dispersal effectiveness more strongly in secondary $(+52.2 \%)$ vs. in primary forest $(+5.0 \%)$, in L. nigrifrons $(+12.9 \%)$ vs. in S. mystax
\end{abstract}

Handling Editor: Joanna M. Setchell

Electronic supplementary material The online version of this article (https://doi.org/10.1007/s10764-0180041-y) contains supplementary material, which is available to authorized users.

Laurence Culot

1culot@rc.unesp.br

1 Laboratório de Primatologia, Departamento de Zoologia, Instituto de Biociências, Universidade Estadual Paulista (UNESP), Rio Claro, SP 13506-900, Brazil

2 Behavioral Biology Unit, Primatology Research Group, University of Liège, 4020 Liège, Belgium

3 Abteilung Verhaltensökologie \& Soziobiologie, Deutsches Primatenzentrum (DPZ),

37077 Göttingen, Germany 
$(+7.9 \%)$ feces, and in larger fecal portions $(+22.1 \%)$ vs. in small-medium ones $(+7.3-$ $7.4 \%$ ). In conclusion, two seed dispersers are more effective than one only in secondary forest, and the magnitude of increase of seed dispersal effectiveness with phase II depends on how the seeds are primarily dispersed.

Keywords Context dependence $\cdot$ Primary and secondary dispersal $\cdot$ Seed burial $\cdot$ Seed survival $\cdot$ Seedling recruitment

\section{Introduction}

Natural regeneration of forests is a complex multistaged process that often starts with primary seed dispersal, which is the initial movement of seeds away from the parent plant. This first step is followed by a chain of postdispersal events and processes, such as secondary seed dispersal (subsequent movement of the seeds, usually to safer sites), seed predation, seed germination, and seedling recruitment (Balcomb and Chapman 2003; Wang and Smith 2002). Primary seed dispersal provides the template for these events and processes (Nathan and Muller-Landau 2000). The type of dispersal vector, dispersal distance, habitat of seed deposition, and seed treatment by the vector are likely to influence the steps following primary dispersal (Beckman and Rogers 2013; Nathan and Muller-Landau 2000; Traveset et al. 2007). Consequently, seed dispersal effectiveness (SDE, Schupp 1993; Schupp et al. 2010) can be viewed as the result of a process in which the primary seed dispersal will determine, directly or indirectly, the final fate of the seeds in the plant regeneration process, through a series of cascading effects in which the outcome of one event defines the context for subsequent events.

Diplochory is defined as the dispersal of seeds by at least two different vectors in two successive steps (or more) called phase I (primary seed dispersal) and phase II (secondary seed dispersal by a mechanism different from that of phase I; Chambers and MacMahon 1994; Vander Wall and Longland 2004). One widespread diplochorous system in the tropics is phase I through primate defecations followed by phase II by dung beetles (Shepherd and Chapman 1998; Vander Wall and Longland 2004; Vulinec 2000). Primates disperse large quantities of seeds, from small to large ones (Chapman 1995), and their defecations attract dung beetles likely to move the seeds horizontally and/or vertically by burying the seeds while building their nests (Andresen and Feer 2005; Culot et al. 2011). In such systems, some characteristics of the defecation pattern, such as habitat of seed deposition, quantity of fecal matter, time of defecation, or seed density, likely influence the probability and the outcome of phase II (Andresen 2001; Andresen and Levey 2004; Beaune et al. 2012; Culot et al. 2009; Santos-Heredia et al. 2010). For instance, seeds dispersed in larger amounts of howler (Alouatta seniculus) dung are more likely to be buried because they attract a higher abundance and diversity of dung beetles (Andresen 2001). Seeds dispersed by tamarins (Saguinus mystax and Leontocebus nigrifrons) in the afternoon are more likely to attract the larger dung beetles of the nocturnal community, consequently increasing their burial probability (Culot et al. 2011). The horizontal movement of seeds during phase II dispersal may reduce the clustering of seedlings (Lawson et al. 2012). Through vertical movement, 
buried seeds may be better protected from predation and in a more suitable environment for germination, but burial may also hinder germination and seedling emergence if seeds are buried too deep (Andresen and Levey 2004; Beaune et al. 2012; Shepherd and Chapman 1998). Although this suggests that phase II dispersal can positively or negatively affect seed germination, seedling recruitment, and survival, the question of whether two dispersers are better than one, i.e., whether overall SDE is increased or decreased with phase II, and whether this is context dependent, has been little examined so far (Culot et al. 2015).

Given that phase II dispersal can change plant fitness, we need to understand better which characteristics of phase I dispersal correlate with the occurrence of phase II dispersal and its specific outcomes. In addition, the outcomes of phase II may vary with habitat because of differences in secondary seed disperser and seed predator communities (Culot et al. 2011; Gallegos et al. 2014) and in abiotic factors (Kupsch et al. 2014). For instance, secondary seed dispersal by ants increased seedling recruitment in secondary forest, but not in primary forest of the Bolivian Andes (Gallegos et al. 2014).

Seed dispersal effectiveness is defined by the product of a quantitative and a qualitative component. The quantitative component is the number of seeds dispersed while the qualitative component is the probability that a dispersed seed will grow into an adult plant (or a seedling, depending on the proxy used; Schupp et al. 2010). Several studies have assessed various aspects of primate seed dispersal effectiveness, but few have considered phase II events when evaluating the qualitative component (e.g., Andresen and Levey 2004; Culot et al. 2015; Lugon et al. 2017). Indeed, the estimation of the qualitative component of dispersal effectiveness has often been restricted to the determination of seed dispersal distances and germination success, while other postdispersal events (secondary dispersal, predation) and seedling recruitment have received less attention ( $c f$. , e.g., Andresen 2001; McConkey and Brockelman 2011; Razafindratsima and Dunham 2014).

Recently, we proposed a method to quantify the effect of phase II dispersal on SDE (Culot et al. 2015). This method is an extension of the SDE concept that enables the formal integration of phase II dispersal when calculating SDE. The extension enables the determination of how much SDE is increased or decreased by phase II dispersal based on the quality components of one-phase $(Q u 1)$ and two-phase $(Q u 2)$ dispersal and the probability of occurrence of phase II dispersal (Pb2; Culot et al. 2015). The magnitude and direction of the effect of a phase II dispersal on SDE (SDE change $_{\text {) }}$ depend on the phase II dispersal probability $(\mathrm{Pb2})$ and the proportional change of dispersal quality from a one-phase dispersal to a two-phase dispersal ((Qu2-Qu1)/ Qu1) (Eq. 1; Culot et al. 2015).

$$
\text { SDEchange }=\frac{P b 2 *(Q u 2-Q u 1)}{Q u 1}
$$

Using model selection and the foregoing method with a system involving phase I dispersal by two primate species living in a mixed-species group and phase II by dung beetles, we aim to understand the causal relationship of the different steps of plant recruitment and how these steps affect the final SDE at a plant community level. More specifically, we aim to 1) determine the characteristics of phase I dispersal that affect 
the probability of phase II dispersal (specifically seed burial); 2) determine direct and indirect effects on seed/seedling survival during the plant recruitment process; and 3) assess if the direction and/or magnitude of phase II dispersal effects on SDE depend on the characteristics of phase I and on forest type.

\section{Methods}

\section{Study Area}

The study took place between 2004 and 2008 at the Estación Biológica Quebrada Blanco (EBQB) located in northeastern Peru $\left(04^{\circ} 21^{\prime} \mathrm{S}, 73^{\circ} 09^{\prime} \mathrm{W}\right)$ on the right bank of the Quebrada Blanco, an affluent of the Río Tahuayo. The 100-ha study area is dominated by primary terra firme rain forest on a slightly undulating terrain interspersed with small swampy areas (Encarnación 1985; Heymann 1995). The study area included the home range of one mixed-species group of tamarins comprising $c a .36$ ha of primary forest and $\mathrm{ca}$. 4 ha of successional forest regenerating from a pasture abandoned in 2000. More details about the study area can be found in Culot et al. (2010).

\section{Study System}

We studied two primate species living in a mixed-species group: 7-15 tamarins (Callitrichidae), including 4-10 mustached tamarins (Saguinus mystax), and 3-6 saddleback tamarins (Leontocebus nigrifrons). Both species exploit a high diversity of fruits (307 species; Culot et al. 2010), but also feed on gums, nectar, invertebrates, and small vertebrates (Heymann et al. 2000; Nickle and Heymann 1996; Smith 2000). Since they spend most of their active time in close interspecific association (Heymann 1990; Heymann and Buchanan-Smith 2000), their role in primary seed dispersal can be evaluated simultaneously. Despite their small size [S. mystax: 380-650 g (Soini and Soini 1982); L. nigrifrons: 240-440 g (Soini and Coppula 1981)], S. mystax and L. nigrifrons handle and disperse through defecation the seeds of 166 plant species, some of which are as large as $2.35 \mathrm{~cm}$ in length, in primary and secondary forests (Culot et al. 2010). They produce small feces with a small amount of fecal matter but almost always ( $\mathrm{ca}$. 95\% of fecal samples) containing intact seeds in generally low density (Knogge and Heymann 2003). Tamarins, being frugivore-insectivores, do not significantly affect germination success and time (Fuzessy et al. 2016). Dung beetles are the main secondary seed dispersers in our system: tamarin feces attract a community of 25 dung beetle species able to bury seeds at a depth ranging from 0.5 to $11 \mathrm{~cm}$ (Culot et al. 2011).

\section{Data Collection}

We collected field data during a total of 28 mo (two 2-mo periods and four 6-mo periods), with each month represented at least twice: June-July 2004, January-February 2005, September 2005-February 2006, June-November 2006, March-August 2007, and December 2007-May 2008 (296 days, 2393 h). 
To record primary seed dispersal events and the location of feeding plants, we followed one mixed-species group of tamarins for 4 days per week, 3-4 weeks per month, from early morning (when they left their sleeping sites) until late afternoon (when they entered a sleeping site). One day per week, we recorded the following information for each defecation: 1) seed disperser species (Leontocebus nigrifrons or Saguinus mystax); 2) seed species; 3) time of defecation (divided into five 2-h periods); 4) number of seeds per defecation (categories: 1, 2, 3, >3); 5) amount of fecal matter (small: fecal matter almost not visible, medium: clear presence of fecal matter and outlines of the seeds visible, and large: seeds hidden by the quantity of fecal matter); 6) habitat of seed deposition (primary or secondary forest); and 7) distance to the nearest conspecific adult plant $(<30 \mathrm{~m}, 30-50 \mathrm{~m}, 51-100 \mathrm{~m},>100 \mathrm{~m})$. We estimated the distance to the nearest conspecific adult plant by mapping the location of all seed dispersal events and of all feeding trees used by the tamarins during the 28-mo study period, which we consider to be a good estimate of the spatial distribution of these adult plant species in the study area. We marked all intact seeds $\geq 0.4 \mathrm{~cm}$ in length found in fresh tamarin defecations by attaching a $25-\mathrm{cm}$ white nylon thread ending with a $5-\mathrm{cm}$ piece of colored and numbered raffia.

To follow seed fate, we checked marked seeds once a week during the first month, on days $4,11,18$, and 25 after deposition, then 1 mo after deposition, then once a month until the end of the study. We used GPS coordinates and a detailed map of the study site to find the exact location of the initial seed deposition. When checking marked seeds, we took note of their state (alive or dead-eaten or husk softened, for example), position in the soil (buried or not), and life stage (seed, germinated seed [defined by the emergence of radicles], or seedling). We did not consider the possible effect of horizontal movements of seeds by dung beetles because its magnitude is negligible in our system (Culot et al. 2009, 2015). We acknowledge the possible effect of burial depth on seed survival. However, variation in burial depth is very low in our system $(71 \%$ of seeds were buried at $<3.5 \mathrm{~cm}$; L. Culot unpubl. data). Therefore, we chose not to include this variable in our analysis for simplicity.

If seeds were alive and had germinated, we cut the thread to avoid interference with seedling emergence. If seeds had been buried, we partly dug them up very carefully to check for germination or predation, and then reburied them at the same depth in the same position. If a buried seed had germinated, we followed the same procedure as for the unburied seeds but did not dig it up again in the following check; instead we waited for seedling emergence. We defined an established seedling as showing leaf-like cotyledons or first leaves (as the distinction was not always possible) (Andresen 2001). More details on the methodology used to mark the seeds in situ and to follow their fate can be found in Electronic Supplementary Material (ESM) Appendix S1 (previously published in Culot et al. 2015 and its Appendix 1).

In total, we marked and followed the fate of 981 seeds belonging to $\geq 105$ plant species (more information on species dispersed can be found in ESM Appendix 2, previously published in Culot et al. 2010, a study carried out on the same group and at the same time as the present study) from 606 defecations. As we marked seeds throughout each study period, the onset and duration of sampling varied between seeds (a minimum of 11 days and a maximum of $3 \mathrm{yr}$. and $10 \mathrm{mo}$ ). We used different subsets of these seeds for each analysis. 


\section{Data Analyses}

We performed all statistical analyses using R 3.1.1 ( R Development Core Team 2014) with $\alpha$ level set at 0.05 .

\section{Which Characteristics of Phase I Dispersal Affect the Probability of Phase II Dispersal?}

To determine the characteristics of phase I that affect phase II, we examined

1) Effect of the primary disperser on the outcome of phase I: We tested whether tamarin species differ in their phase I pattern (habitat of seed deposition, distance to nearest conspecific plant, time of defecation, number of seeds per feces, and quantity of fecal matter) using $\chi^{2}$ tests with randomization on our almost complete dataset $(N=797$ unambiguously identified seeds for the test on distance to nearest conspecific, and $N=590$ feces for which tamarin species is known for the test of the other variables) using the coin package (Hothorn et al. 2008).

2) Effect of the outcome of phase I on phase II probability: We tested the effect of phase I on seed burial probability using our complete dataset and a generalized linear model (binomial distribution), with the tamarin species, habitat, number of seeds per feces, quantity of fecal matter, and the time of defecation as predictors. We tested possible multicollinearity between predictor variables using the $\mathrm{HH}$ package (Heiberger 2017) and considered a threshold of VIF $<5$ as recommended by McNair et al. (2012). As all predictors had VIF < 5, we included all variables in the model. We used the Akaike information criterion (AIC) to determine the best fit models (Akaike 1973; Johnson and Omland 2004). We did not consider the distance from the nearest conspecific adult plant because there is no reason to predict that it influences dung beetle activity.

\section{Determining Direct and Indirect Effects on Seed/Seedling Survival During the Plant Recruitment Process}

To determine the variables affecting the plant recruitment process, we considered the steps from the primary dispersal phase to $1-\mathrm{yr}$ seed/seedling survival. We used time-to-event analyses (also called survival analyses) in which the response variable is the number of days elapsed until the event occurs (germination, seedling emergence, death). Time-to-event analyses allow for the use of exact and censored data. Exact data are those for which we know exactly when the event occurred (germination, seedling emergence, or death in our case). When we could not continuouosly checked the seeds (between two field sessions) and the event occurred during this interval, we estimated the time of the event as the mean number of days elapsed since the last check before the start of the interval and considered these data as exact data. Right-censored data are data for which we 
know that the event did not occur within the observation time. We tested the effect of phase I and phase II (seed burial) on seed germination, seedling emergence, and seed/seedling survival on a subset of unambiguously identified seeds for which seed fate was followed for $\geq 1$ yr. $(N=503)$. We ran Cox proportional hazard models with feces as frailty factor (i.e., random factor) as suggested by McNair et al. (2012), using the survival package (Therneau 2014). We used AIC to determine the best fit models (Akaike 1973; Johnson and Omland 2004). We considered tamarin species, dispersal habitat, number of seeds per feces, quantity of fecal matter, time of defecation, distance to the nearest conspecific, seed burial, and the interaction between habitat and seed burial as fixed factors with germination as response variable. When our response variable was seedling emergence or seed/seedling survival, we added germination as a fixed effect; when our response variable was seed/seedling survival, we added germination and seedling survival as fixed effects. We considered only the interaction of factors that we judged to have biological relevance. We tested possible multicollinearity between predictor variables using the $\mathrm{HH}$ package (Heiberger 2017) and considered a threshold of $\mathrm{VIF}<5$ as McNair et al. (2012) recommended. As all predictors had VIF $<5$, we included all variables in the model.

\section{Assessing How Strongly Phase II Dispersal Affects SDE in Different Primary Dispersal Contexts}

We used the phase II effect landscape to visualize the changes of SDE with seed burial according to different primary dispersal contexts (for more details about this method see Culot et al. 2015). The magnitude and direction of the effect of phase II dispersal on SDE depends on the phase II probability $(\mathrm{Pb} 2)$ and the proportional change of dispersal quality from one-phase dispersal to two-phase dispersal ( $(Q u 2-$ Qu1)/Qu1; Culot et al. 2015). Our measure of phase II is the occurrence of seed burial (Pb2), while $\mathrm{Pbl}$ is the proportion of seeds not buried after 4 days (Culot et al. 2015). We defined the quality component when the seeds are not buried (Qu1) or buried (Qu2) as the seed/seedling survival probabilities. To compare seed/seedling survival of all species at the same life stage, we considered the survival of seeds and seedlings within 1 mo after the last observed seedling emergence of each species. How much SDE of one-phase dispersal changes compared to dispersal in two phases is given by multiplying the two components of the phase II effect landscape and multiplying this result by 100 to obtain the percentage of increase or decrease of SDE, the quantity of seeds primarily dispersed remaining constant (Culot et al. 2015). Using this approach, we built the phase II effect landscape for the plant community in different primary dispersal contexts shown to significantly influence seed burial probability. We visualized which phase I characteristics increased SDE through enhancing seed burial probability, as well as the magnitude of the increase. We used the same subset of seeds as for the time-to-event analysis $(N=503)$.

Data Availability The datasets during and/or analyzed during the current study are available from the corresponding author on reasonable request. 


\section{Ethical Note}

We conducted this research under permit from the Instituto Nacional de Recursos Naturales (INRENA, Peru; authorizations 011-2005-INRENA-IFFS-DCB, 0712005- INRENA-IFFS-DCB，059-2006-INRENA-IFFS-DCB, and 114-2007INRENA-IFFS-DCB). We declare no conflict of interest.

\section{Results}

\section{Which Characteristics of Phase I Dispersal Affect the Probability of Phase II Dispersal?}

Effect of the Primary Disperser on the Outcome of Phase I The comparison of the defecation pattern of Leontocebus nigrifrons and Saguinus mystax showed that the two tamarin species differed in the number of seeds per feces $\left(N=590, \chi^{2}=15.39, \mathrm{df}=3\right.$, $P=0.001)$ and in the quantity of fecal matter produced $\left(N=590, \chi^{2}=25.69, \mathrm{df}=2\right.$, $P=0.000003) . S$. mystax produced more feces with one seed and more than three seeds and produced a higher proportion of feces with a small quantity of fecal matter than L. nigrifrons. The habitat of seed deposition and the time of defecation did not significantly differ between species (habitat: $N=590, \chi^{2}=2.64, \mathrm{df}=1, P=0.104$; time of defecation: $N=590, \chi^{2}=7.77, \mathrm{df}=4, P=0.100$ ) (Fig. 1).

A
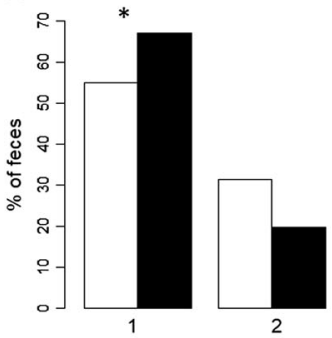

Number of seeds

C

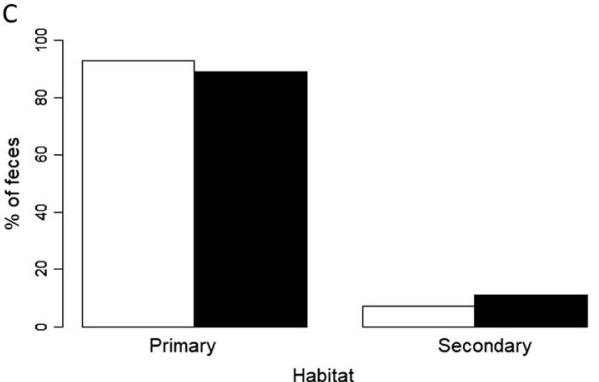

B
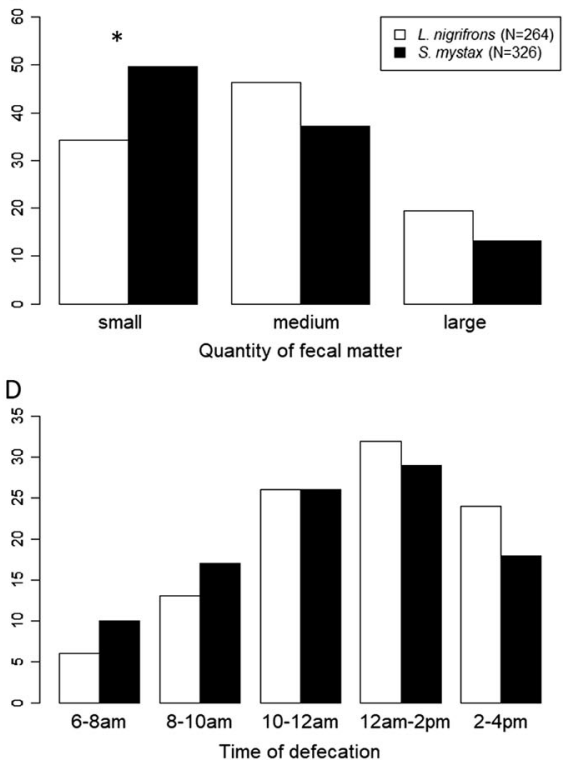

Fig. 1 Characteristics of phase I dispersal by tamarins, Saguinus mystax and Leontocebus nigrifrons, in the Estación Biológica Quebrada Blanco (Peru) in 2004-2008. Percentage of feces $(N=590)$ a with one, two, three, and more than three seeds; $\mathbf{b}$ with small, medium, and large quantity of fecal matter; $\mathbf{c}$ defecated in primary and secondary forests; and $\mathbf{d}$ defecated between 06:00 and 08:00 h, 08:00 and 10:00 h, 10:00 and 12:00 h, 12:00 and 14:00 h, and 14:00 and 16:00 h. Asterisks show significant differences $(P<0.05)$ between L. nigrifrons and S. mystax (see Data Analyses). 
Effect of the Outcome of Phase I on Phase II Probability Two equally plausible best fitted models explained seed burial probability. The first and simplest one contained tamarin species, quantity of fecal matter, and number of seeds per feces as explanatory (and significant) variables (Table I). Seeds dispersed by Leontocebus nigrifrons were more likely to be buried by dung beetles than seeds dispersed by Saguinus mystax. Seeds dispersed in a large quantity of fecal matter were more likely to be buried than seeds dispersed in medium or small quantities of fecal matter. When only two seeds were dispersed in one feces, they were significantly more likely to be buried than seeds dispersed singly, or with more seeds (Fig. 2).

\section{Determining Direct and Indirect Effects on Seed/Seedling Survival During the Plant Recruitment Process}

Overall, $22 \%(112 / 503)$ of seeds survived for $\geq 1$ yr., either as seed (12\%) or as seedling (88\%). Of the 391 seeds/seedlings that did not survive, $81 \%$ (317/391) died in the seed stage, $8 \%$ (32/391) shortly after germination and $11 \%(42 / 391)$ at the seedling stage. Most mortality (52\%) occurred within the first month after dispersal. Causes of seed mortality were predation by vertebrates or invertebrates in $80 \%$ of cases (278/349), followed by fungus or parasites $(13 \%, 44 / 349)$ and destruction by tree and branch fall $(8 \%, 27 / 349)$. Causes of seedling mortality were desiccation or herbivory in $93 \%$ of cases $(39 / 42)$ followed by destruction by tree fall $(7 \%, 3 / 42)$.

Seed germination was best explained by the model without tamarins (Table II). Only habitat and number of seeds per feces exerted a significant effect on seed germination (ESM Appendix 3): seeds dispersed in primary forest and in feces with more than three seeds were less likely to germinate than seeds dispersed in secondary forest $(P=$ 0.0001 ) and in feces with a lower density of seeds (feces with one vs. more than three seeds: $P=0.01$, feces with two seeds vs. more than three seeds, $P=0.04$, and feces with three seeds vs. more than three seeds: $P<0.049$ ) (Fig. 3a, b).

Table I AIC-based model selection based on generalized linear model analyses with a set of candidate models for predicting seed burial probability considering tamarin species (Saguinus mystax and Leontocebus nigrifrons), quantity of fecal matter, number of seeds per feces, habitat of seed deposition, and time of defecation (td) as explanatory variables $(N=956)$ in the Estación Biológica Quebrada Blanco (Peru) in 2004 2008

\begin{tabular}{lllll}
\hline Model rank & Model & AIC & $\Delta$ AIC & AICW \\
\hline $\mathbf{1}$ & Tamarin + number of seeds + quantity of fecal matter & $\mathbf{1 0 2 0 . 6}$ & $\mathbf{0}$ & $\mathbf{0 . 6 1}$ \\
$\mathbf{2}$ & Tamarin + number of seeds + quantity of fecal matter + habitat & $\mathbf{1 0 2 2 . 2}$ & $\mathbf{1 . 6}$ & $\mathbf{0 . 2 6}$ \\
3 & Tamarin + number of seeds & 1024.0 & 3.4 & 0.11 \\
4 & Tamarin + number of seeds + quantity of fecal matter + time of & 1028.0 & 7.4 & 0.01 \\
& $\quad$ defecation & & & \\
5 & Tamarin + number of seeds + quantity of fecal matter + habitat + & 1029.7 & 9.1 & 0.01 \\
& time of defecation & & & \\
\hline
\end{tabular}

The models are ranked from best to worst fit according to the $\triangle \mathrm{AIC}$ and AIC weights (AICw) are shown. Only the five best models are shown. Models with $\triangle \mathrm{AIC}<2$ are equally plausible. Variables with significant effect $(P<0.05)$ in the models are in bold type 

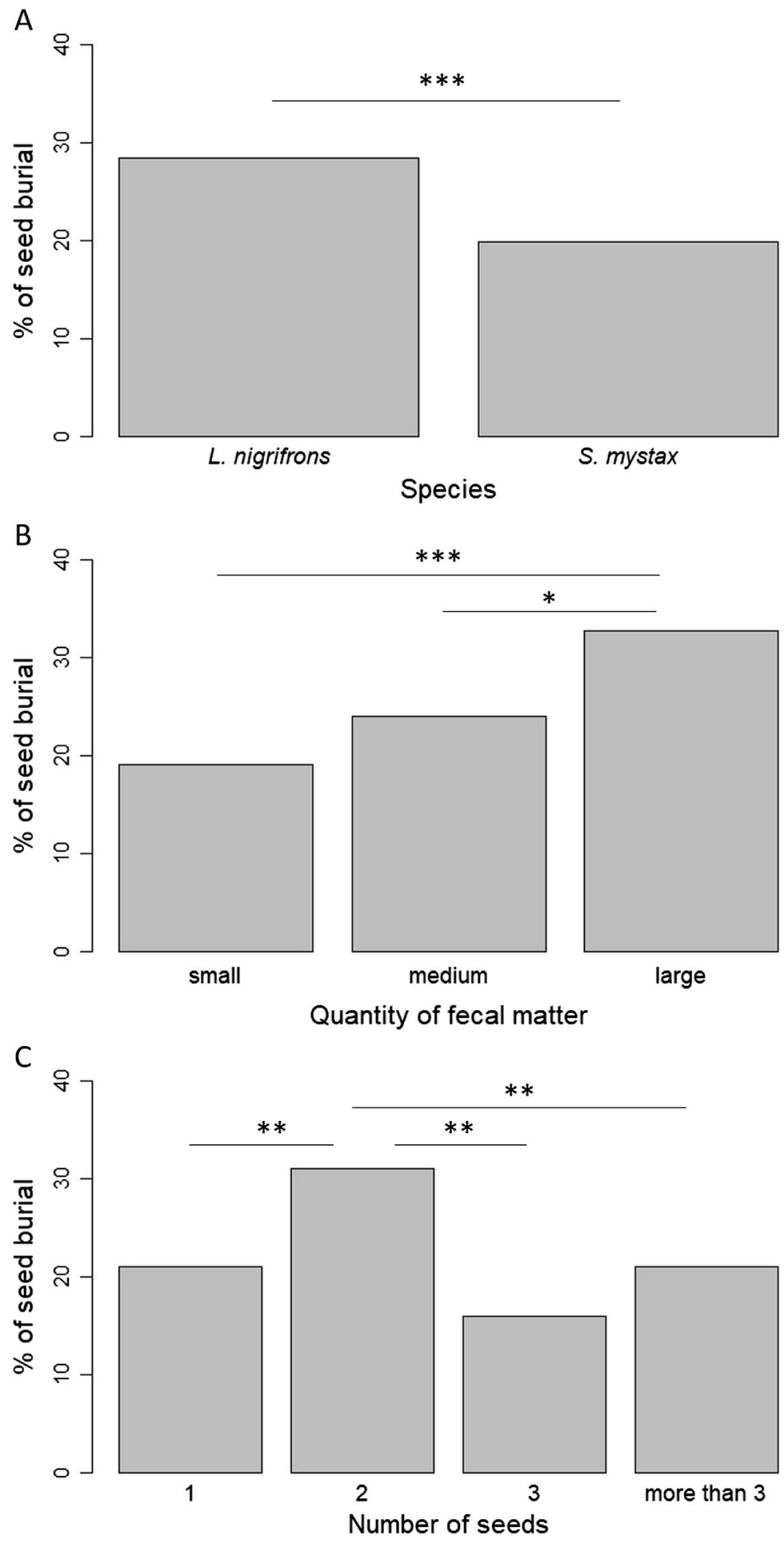
Fig. 2 Seed burial probability according to the characteristics of phase I dispersal in the Estación Biológica Quebrada Blanco (Peru) in 2004-2008. Percentage of seeds buried $(N=956)$ according to a the tamarin species (Leontocebus nigrifrons and Saguinus mystax), b the quantity of fecal matter, and $\mathbf{c}$ the number of seeds per feces. Asterisks show significant differences $(* P<0.05, * * P<0.01, * * * P<0.001)$

Seedling emergence was best explained by the model without the number of seeds (Table II). Tamarin species, habitat, and time of defecation had significant effects on seedling emergence (ESM Appendix 3): seeds dispersed by Leontocebus nigrifrons $(P=0.003)$, in secondary forest $(P=0.01)$, very early in the morning (before 08:00 h) $(P=0.017)$, or in the afternoon $(P=0.02$ and $P=0.0009)$ were more likely to emerge as seedlings than seeds dispersed by Saguinus mystax (Fig. 3c), in the primary forest (Fig. 3d) or later in the morning.

Seed/seedling survival was best explained by the model containing all the explanatory variables (Table II). However, only seed burial and the interaction between habitat and seed burial had a significant effect on seed/seedling survival (ESM Appendix 3). Seeds that had been buried by dung beetles were more likely to survive than seeds not buried (Fig. 3e) but the interaction term showed that this effect was significant only in secondary forest $(P=0.001)$ (Fig. 3f).

\section{Assessing How Strongly Phase II Dispersal Affects SDE in Different Primary Dispersal Contexts}

Given our previous results we built the phase II effect landscape according to 1) the tamarin species, 2) the quantity of fecal matter, and 3) the habitat. SDE increased by $12.9 \%$ with phase II when seeds were dispersed primarily by Leontocebus nigrifrons and by $7.9 \%$ when dispersed primarily by Saguinus mystax. Most of the difference was explained by differences in seed burial probability (0.25 vs. 0.19 ; Fig. 4a). Seeds dispersed in small amounts of fecal

Table II Best fit models of the effects of the characteristics of phase I dispersal by Saguinus mystax and Leontocebus nigrifrons and seed burial by dung beetles on seed germination, seedling emergence, and seed/seedling survival in the Estación Biológica Quebrada Blanco (Peru) in 2004-2008

\begin{tabular}{llc}
\hline Response variable & Model & AIC \\
\hline Seed germination & $\begin{array}{c}\text { Quantity of fecal matter + habitat + number of seeds + time of defecation } \\
\text { + distance + seed burial + habitat:seed burial }\end{array}$ & 1690.1 \\
Seedling emergence & $\begin{array}{c}\text { Tamarin + quantity of fecal matter + habitat + time of defecation } \\
\text { + distance + seed burial + habitat:seed burial }\end{array}$ & 1354.3 \\
Seed/seedling survival & $\begin{array}{l}\text { Tamarin + quantity of fecal matter + habitat + number of seeds } \\
\text { + time of defecation + distance + seed burial + habitat:seed burial }\end{array}$ & 4235.1 \\
\hline
\end{tabular}

The models are based on the Cox proportional hazard models analyses with feces as a frailty factor $(N=503)$. Explanatory variables in our models were tamarin species, quantity of fecal matter, number of seeds per feces, habitat of seed deposition, time of defecation, distance to the nearest conspecific adult plant, seed burial, and the interaction between habitat and seed burial. Only the most plausible models are presented here. Variables with significant effect in the models are in bold type 

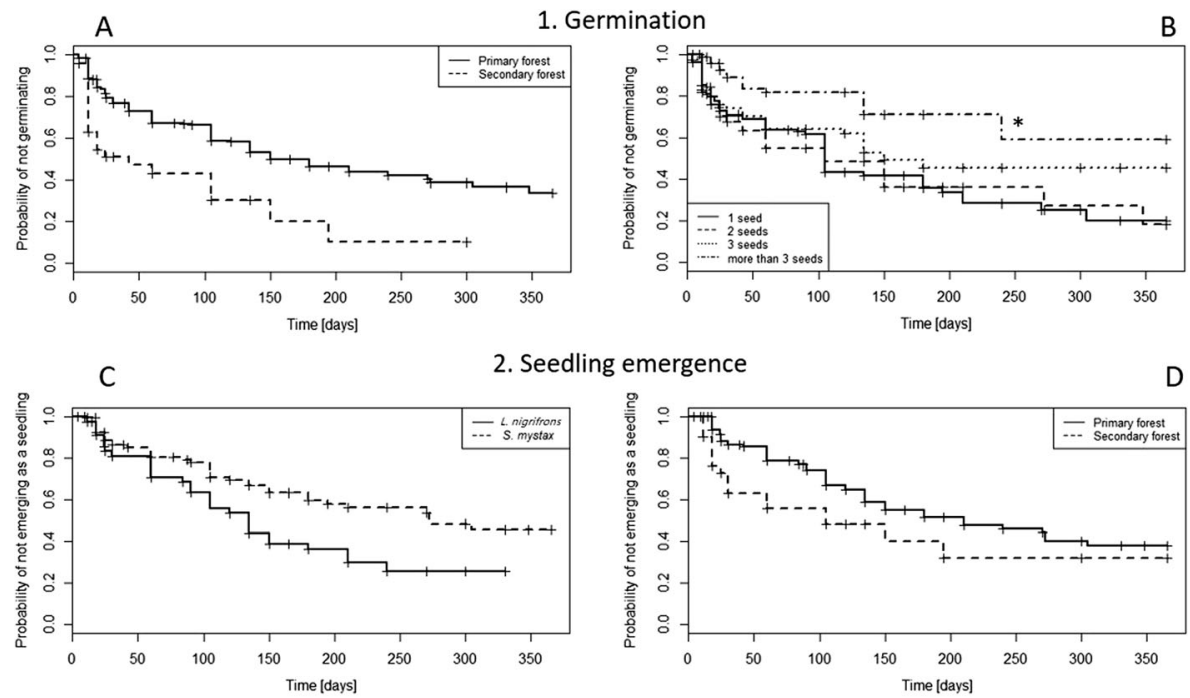

$\mathrm{E}$

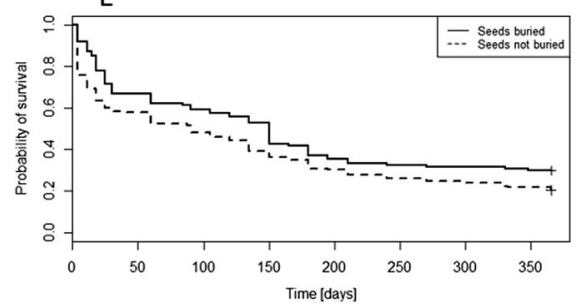

Fig. 3 Kaplan-Meier curves showing the effects of the characteristics of phase I dispersal by Saguinus mystax and Leontocebus nigrifrons and seed burial by dung beetles on seed germination, seedling emergence, and seed/seedling survival in the Estación Biológica Quebrada Blanco (Peru) in 2004-2008. The Kaplan-Meier estimates of survivor functions show the probability of 1) not germinating according to a the habitat of seed deposition (primary forest: $N=456$, secondary forest: $N=47$ ) and $\mathbf{b}$ the number of seeds per feces ( 1 seed: $N=200,2$ seeds: $N=129,3$ seeds: $N=77,>3$ seeds: $N=97$ ); 2) not emerging as a seedling according to $\mathbf{c}$ the tamarin species (L. nigrifrons: $N=202, S$. mystax: $N=301$ ) and d habitat (primary forest: $N=456$, secondary forest: $N=47$ ); and 3) seed/seedling survival according to e seed burial (seeds buried: $N=108$, seeds not buried: $N=395$ ) and $\mathbf{f}$ seed burial in primary and secondary forest (seeds not buried vs. buried in primary forest: $N=359$ vs. $N=97$ and in secondary forest: $N=36$ vs. $N=11$ ) for an observation time of 1 yr. Censored data are denoted by crosses. Asterisks indicate which curve is significantly different from all others (see Data Analyses).

matter were less likely to be buried than seeds in medium or large quantity of fecal matter. In addition, the proportional change of dispersal quality with phase II dispersal was stronger for seeds dispersed in large quantity of fecal matter than in medium and small quantities. This difference explained the larger increase of SDE with phase II for seeds in large $(+22.1 \%)$ than in medium $(+7.4 \%)$ or small $(+7.3 \%)$ quantities of fecal matter (Fig. 4b). Seed burial increased SDE in primary and secondary forests but the effect was much stronger in secondary forest $(+52.2 \%)$ than in primary forest $(+5.0 \%)$ owing to the higher proportional change of seed dispersal quality. The probability of seed burial was very similar in both habitats (primary forest: 0.21 , secondary forest: 0.23; Fig. 4c). 


\section{Discussion}

We found that the characteristics of primate defecation affect seed fate both directly, by affecting seed germination, seedling emergence, and seed/seedling survival, and indirectly, by affecting seed burial probability by dung beetles. Our study showed that, at a community level, two dispersers were better than one in secondary forest, but not in primary forest, matching results for secondary seed dispersal by ants in the Bolivian Andes (Gallegos et al. 2014). Seed burial by dung beetles directly enhanced seed/seedling survival in secondary forest, increasing SDE much more markedly than in primary forest.

We compared two primary disperser species of similar size and diet (Culot et al. 2010; Peres 1993), which enables the comparison of the seed fate of the same plant community when dispersed by two frugivore species. The significant direct effect of tamarin species on seed burial reflects their differences in defecation pattern: Leontocebus nigrifrons dispersed seeds in large amounts of fecal matter more often than Saguinus mystax, consequently increasing seed burial probability and allowing a gretear increase in SDE with seed burial compared to seeds dispersed by S. mystax (12.9 vs. $7.9 \%)$. Unmeasured characteristics of phase I dispersal linked to the tamarin species, such as the stronger odor of L. nigrifrons' feces, for example (L. Culot pers. $o b s$.), could also have influenced attractiveness to dung beetles and thus seed burial probability. Many studies have shown that increasing the amount of dung increases seed burial probability by dung beetles (Andresen 2001; Andresen and Levey 2004; Culot et al. 2009, 2011; Errouissi et al. 2004; Santos-Heredia et al. 2010). Here, we show that even small differences in defecation patterns, such as a small variation in the amount of fecal matter, can increase seed burial probability and therefore modify seed fate. The increase in SDE of seeds dispersed in large amounts of fecal matter was caused by the higher proportional change of dispersal quality compared to seeds dispersed in smaller amounts of fecal matter. This might be due to the larger supply of nutrients provided by larger amounts of dung, possibly enhancing seedling emergence (Traveset et al. 2001), and therefore also seed/seedling survival. We hypothesize that fecal matter remains longer around buried than unburied seeds exposed to desiccation and rainfall, and that this would enable buried seeds to benefit more than unburied seeds from the nutrients provided by the feces for seedling emergence. This hypothesis needs to be tested.

Other factors linked to the defecation pattern indirectly influenced seedling survival through germination and/or seedling emergence. Low density of seeds (one to three seeds) deposited in primary forest increased germination probability. Single-seed defecation is a very frequent event in tamarins, and the majority of their feces ( $c a$. 75\%-85\%) contain only one or two seeds (Knogge and Heymann 2003). Single seeds are typically defecated by small frugivores (Knogge and Heymann 2003), regurgitated by birds (Stiles 2000), or spat out by cheek-pouched primates (Gross-Camp and Kaplin 2011). Our results corroborate those of previous studies showing that seeds spat or defecated singly experience higher seedling establishment rate than clumped seeds (Balcomb and Chapman 2003; Gross-Camp and Kaplin 2011) and stress the importance of small frugivores that are able to disperse large seeds, for forest regeneration. Here again, small differences in the number of seeds defecated together can have a significant effect on seed fate. 

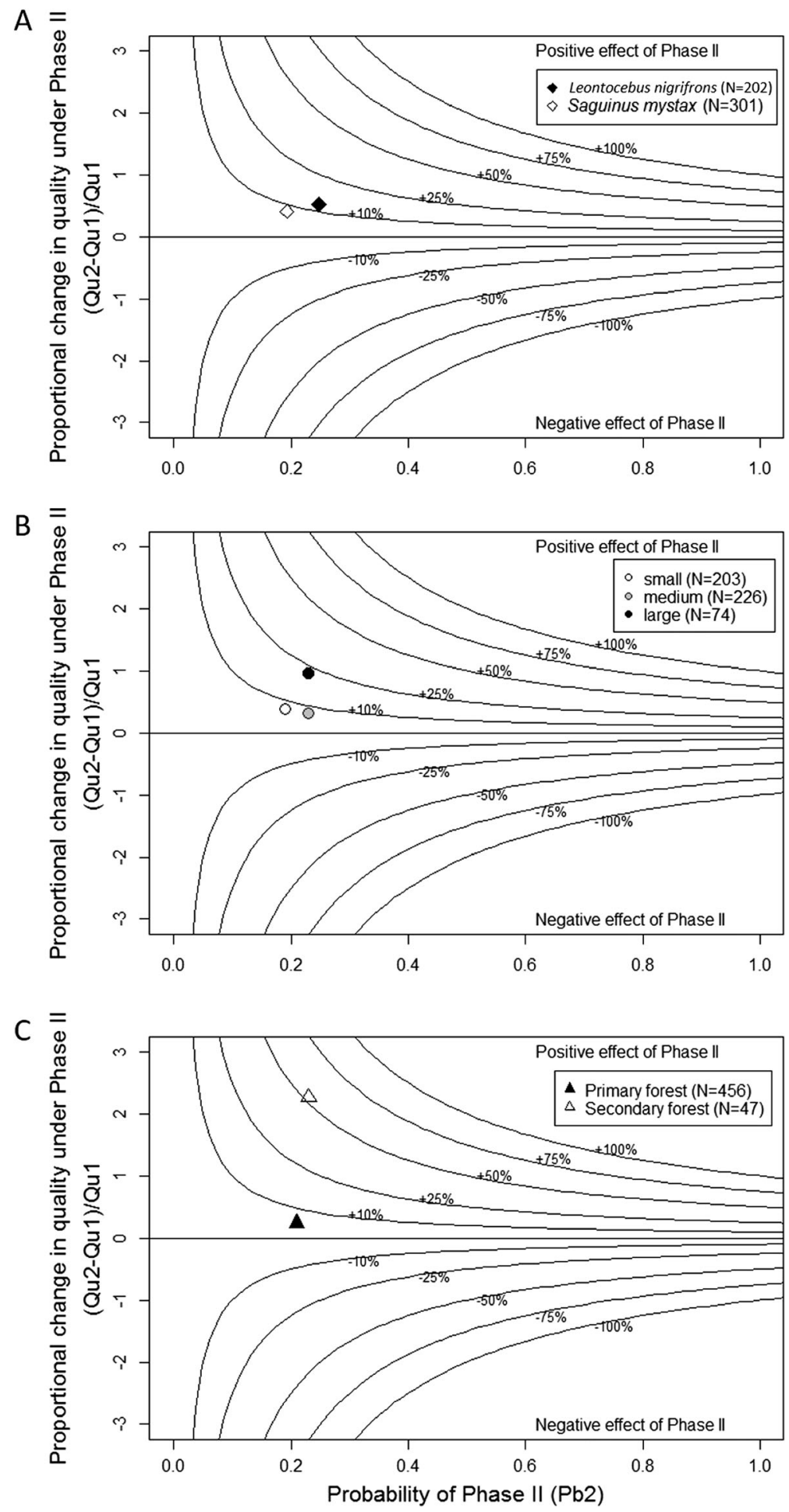
Fig. 4 Phase II effect landscape for the plant community dispersed by Saguinus mystax and Leontocebus nigrifrons between 2004 and 2008 at the Estación Biológica Quebrada Blanco (Peru) according to different dispersal contexts: a the tamarin species, $\mathbf{b}$ the quantity of fecal matter, and $\mathbf{c}$ the habitat. Changes in SDE due to seed burial are obtained by multiplying the probability of seed burial $(\mathrm{Pb2})$ by the proportional change of seed/seedling survival with a phase II dispersal $((Q u 2-Q u 1) / Q u 1)$. Isoclines represent all combinations of $x$ and $y$-axes values that yield the same change of SDE with a phase II dispersal. Values assigned to isoclines are the changes of SDE multiplied by 100 in order to obtain percentages of increase or decrease of SDE. More details about the method can be found in Culot et al. (2015).

We found that seed burial by dung beetles significantly increased seed/seedling survival in secondary forest (SDE increased by 52\%), but not in primary forest (SDE increased by 5\%). The secondary forest at the study site was relatively young at the time of our field work (4-8 yr), with a much lower canopy height (mean \pm SD: $18.1 \pm$ $2.7 \mathrm{~m}$ in 2011; Kupsch et al. 2014) compared to the primary forest (24.5 $\pm 3.1 \mathrm{~m}$; Kupsch et al. 2014), likely resulting in a drier environment (Heymann et al. 2014; Kupsch et al. 2014). The risk of desiccation might have been a limiting factor for seed/seedling survival in secondary forest and seed burial could have reduced this risk.

The effect of seed burial varies between plant species both in direction and magnitude (Culot et al. 2015). Whereas some species require light exposure to germinate, others show germination capacity at greater soil depth or suffer from light inhibition on the soil surface (Pons 2000). In addition to differences in light sensitivity, seed size (Foster and Janson 1985; Leishman et al. 2000) and functional morphology of seedlings (Kitajima and Fenner 2000) can be responsible for species-specific responses to seed burial (Andresen and Feer 2005). It would be interesting to test the responses of different plant species along the chain of seed dispersal and postdispersal events. Unfortunately, the diversity of seeds dispersed by our two study tamarin species was so high that it was not possible to select a subsample of plant species with a sufficient number of seeds to test this. However, the study at a community level reveals some general patterns. We found that seed burial has a positive effect on seed/seedling survival but that this effect is much stronger in secondary than in primary forest. Our study showed that a small quantity of seeds and a large amount of dung increased SDE in primary and secondary forests through distinct ways. In both habitats, a small number of seeds enabled better germination, and consequently may have increased survival through the reduction of competition. In secondary forest, a larger quantity of fecal matter enhanced seed burial that directly increased survival.

Our finding that phases I and II make differential contributions to plant survival leads to the question of whether and how much plants can influence the outcome of phase II and thus whether this may influence the evolution of diplochorous seed dispersal systems. Fruit and seed traits affect handling by primary dispersers (Dowsett-Lemaire 1988; Gautier-Hion et al. 1985; Gross-Camp and Kaplin 2011; Levey 1987; Stevenson 2011). Secondary dispersers such as dung beetles are influenced by seed traits like size (Andresen and Feer 2005; Shepherd and Chapman 1998) and shape (Culot et al. 2011) but are also apparently influenced by qualitative and quantitative characteristics of faeces containing the seeds (Andresen 2001; Hanski and Cambefort 1991). Seed size selection by primates and dung beetles should occur in the same direction because smaller seeds are both easier to swallow and to incorporate into dung balls. In contrast, the selection of seed shape might occur in opposite directions: 
more elongated seeds are easier to swallow (Chapman and Russo 2006) while more spherical seeds are more likely to be incorporated into dung balls (Culot et al. 2011). To test whether plants can influence phase II dispersal, we would need to test whether fruit and seed traits and dispersal outcomes vary with the communities of primary and secondary seed dispersers. This is a challenging task, particularly as the effect size might be extremely small, but over the life time of a plant with eventually millions to billions of seeds produced be sufficiently large to provide a fitness benefit.

In conclusion, our study highlights the necessity of considering the postdispersal fate of seeds dispersed by primates to understand better their role in this important ecological process. It is well known that primates disperse large number of seeds of a high diversity of plants and in different habitats, but we still need data on the chain of postdispersal events to assess their effectiveness. Data on postdispersal seed fate would also enable the more accurate determination of the effects of local primate extinctions on future plant vegetation (Culot et al. 2017) and a better understanding of ecosystem functioning as a whole.

Acknowledgements We are grateful to our field assistant, Jeisen Shahuano Tello, for his help on the field, and to Ricardo Zárate and Carlos Amasifuen for the identification of plant species and forest characterization. We thank Ellen Andresen and three anonymous reviewers for their useful comments on a previous version of the article, as well as the editor-in-chief, Dr. Joanna M. Setchell, and the guest editor, Dr. Onja H. Razafindratsima. This study was made possible thanks to a grant from FRIA (Fonds pour la formation à la recherche dans l'industrie et dans l'agriculture) and FNRS (Fonds National de la Recherche Scientifique), Belgium, to L. Culot. L. Culot was financed by a FAPESP (Fundação de Amparo à Pesquisa do Estado de São Paulo) grant during the writing of this article (2014/14739-0). We thank Drs. Yamato Tsuji, Hiroki Sato, and Onja H. Razafindratsima for inviting us to contribute to this special issue.

\section{References}

Akaike, H. (1973). Information theory as an extension of the maximum likelihood principle. In B. N. Petrov \& F. Csaki (Eds.), Second international symposium on information theory (pp. 267-281). Budapest: Akademiai Kiado.

Andresen, E. (2001). Effects of dung presence, dung amount and secondary dispersal by dung beetles on the fate of Micropholis guyanensis (Sapotaceae) seeds in Central Amazonia. Journal of Tropical Ecology, $17(1), 61-78$.

Andresen, E., \& Feer, F. (2005). The role of dung beetles as secondary seed dispersers and their effect on plant regeneration in tropical rainforests (432, Trans.). In P.-M. Forget, J. E. Lambert, P. E. Hulme, \& S. B. Vander Wall (Eds.), Seed fate: Predation, dispersal and seedling establishment (pp. 331-349). Wallingford: $\mathrm{CAB}$ International.

Andresen, E., \& Levey, D. J. (2004). Effects of dung and seed size on secondary dispersal, seed predation, and seedling establishment of rain forest trees. Oecologia, 139, 45-54.

Balcomb, S. R., \& Chapman, C. A. (2003). Bridging the gap: Influence of seed deposition on seedling recruitment in a primate-tree interaction. Ecological Monographs, 73(4), 625-642.

Beaune, D., Bollache, L., Bretagnolle, F., \& Fruth, B. (2012). Dung beetles are critical in preventing postdispersal seed removal by rodents in Congo rain forest. Journal of Tropical Ecology, 28(5), 507-510.

Beckman, N. G., \& Rogers, H. S. (2013). Consequences of seed dispersal for plant recruitment in tropical forests: Interactions within the seedscape. Biotropica, 45(6), 666-681.

Chambers, J. C., \& MacMahon, J. A. (1994). A day in the life of a seed: Movements and fates of seeds and their implications for natural and managed systems. Annual Review of Ecology and Systematics, 25, 263292.

Chapman, C. A. (1995). Primate seed dispersal: Coevolution and conservation implications. Evolutionary Anthropology, 4(3), 74-82. 
Chapman, C. A., \& Russo, S. E. (2006). Primate seed dispersal: Linking behavioral ecology with forest community structure. In C. J. Campbell, A. Fuentes, K. C. MacKinnon, M. Panger, \& K. Bearder (Eds.), Primates in perspective (pp. 510-525). Oxford: Oxford University Press.

Culot, L., Bello, C., Batista, J. L. F., do Couto, H. T. Z., \& Galetti, M. (2017). Synergistic effects of seed disperser and predator loss on recruitment success and long-term consequences for carbon stocks in tropical rainforests. Scientific Reports, 7(1), 7662.

Culot, L., Huynen, M.-C., Gérard, P., \& Heymann, E. W. (2009). Short-term post-dispersal fate of seeds defecated by two small primate species (Saguinus mystax and Saguinus fuscicollis) in the Amazonian forest of Peru. Journal of Tropical Ecology, 25(3), 229-238.

Culot, L., Huynen, M.-C., \& Heymann, E. W. (2015). Partitioning the relative contribution of one-phase and two-phase seed dispersal when evaluating seed dispersal effectiveness. Methods in Ecology and Evolution, 6(2), 178-186.

Culot, L., Mann, D. J., Muñoz Lazo, F. J. J., Huynen, M.-C., \& Heymann, E. W. (2011). Tamarins and dung beetles: An efficient diplochorous dispersal system for forest regeneration. Biotropica, 43(1), 84-92.

Culot, L., Muñoz Lazo, F. J. J., Huynen, M.-C., Poncin, P., \& Heymann, E. W. (2010). Seasonal variation in seed dispersal by tamarins alters seed rain in a secondary rainforest. International Journal of Primatology, 31(4), 553-569.

Development Core Team, R. (2014). R: A language and environment for statistical computing. Vienna: R Foundation for Statistical Computing http:/www.R-project.org/.

Dowsett-Lemaire, F. (1988). Fruit choice and seed dissemination by birds and mammals in the evergreen forests of upland Malawi. Terre et Vie, 43, 251-285.

Encarnación, F. (1985). Introducción a la flora y vegetación de la Amazonía peruana: Estado actual de los estudios, medio natural y ensayo de una clave de determinación de las formaciones vegetales en la llanura amazónica. Candollea, 40, 237-252.

Errouissi, F., Haloti, S., Jay-Robert, P., Janati-Idrissi, A., \& Lumaret, J. (2004). Effects of the attractiveness for dung beetles of dung pat origin and size along a climatic gradient. Environmental Entomology, 33(1), 45-53.

Foster, S. A., \& Janson, C. H. (1985). The relationship between seed size and establishment conditions in tropical woody plants. Ecology, 66(3), 773-780.

Fuzessy, L. F., Cornelissen, T. G., Janson, C., \& Silveira, F. A. O. (2016). How do primates affect seed germination? A meta-analysis of gut passage effects on neotropical plants. Oikos, 125(8), 1069-1080.

Gallegos, S. C., Hensen, I., \& Schleuning, M. (2014). Secondary dispersal by ants promotes forest regeneration after deforestation. Journal of Ecology, 102(3), 659-666.

Gautier-Hion, A., Duplantier, J. M., Quris, R., Feer, F., Sourd, C., et al (1985). Fruit characters as a basis of fruit choice and seed dispersal in a tropical forest vertebrate community. Oecologia, 65(3), 324-337.

Gross-Camp, N., \& Kaplin, B. A. (2011). Differential seed handling by two African primates affects seed fate and establishment of large-seeded trees. Acta Oecologica, 37(6), 578-586.

Hanski, I., \& Cambefort, Y. (1991). Dung beetle ecology. Princeton: Princeton University Press.

Heiberger, R. M. (2017). HH: Statistical analysis and data display: Heiberger and Holland. R package version, 3, 1-34 https://CRAN.R-project.org/package $=$ HH.

Heymann, E. W. (1990). Interspecific relations in a mixed-species troop of moustached tamarins, Saguinus mystax, and saddle-back tamarins, Saguinus fuscicollis (Platyrrhini:Callitrichidae), at the Rio Blanco, Peruvian Amazonia. American Journal of Primatology, 21(2), 115-127.

Heymann, E. W. (1995). Sleeping habits of tamarins, Saguinus mystax and Saguinus fuscicollis (Mammalia; Primates; Callitrichidae), in north-eastern Peru. Journal of Zoology, 237(2), 211-226.

Heymann, E. W., \& Buchanan-Smith, H. M. (2000). The behavioural ecology of mixed-species troops of callitrichine primates. Biological Reviews, 75(2), 169-190.

Heymann, E. W., Knogge, C., \& Tirado Herrera, E. R. (2000). Vertebrate predation by sympatric tamarins, Saguinus mystax and Saguinus fuscicollis. American Journal of Primatology, 51(2), 153-158.

Heymann, E. W., Wörner, L. L. B., Ziegenhagen, B., \& Bialozyt, R. (2014). Research trails affect the abundance of an epiphytic tropical bromeliad. Biotropica, 46, 166-169.

Hothorn, T., Hornik, K., van de Wiel, M. A., \& Zeileis, A. (2008). Implementing a class of permutation tests: The coin package. Journal of Statistical Software, 28(8), 1-23.

Johnson, J. B., \& Omland, K. S. (2004). Model selection in ecology and evolution. Trends in Ecology \& Evolution, 19(2), 101-108.

Kitajima, K., \& Fenner, M. (2000). Ecology of seedling regeneration. In M. Fenner (Ed.), Seeds: The ecology of regeneration in plant communities (pp. 331-359). Wallingford: CAB International.

Knogge, C., \& Heymann, E. W. (2003). Seed dispersal by sympatric tamarins, Saguinus mystax and Saguinus fuscicollis: Diversity and characteristics of plant species. Folia Primatologica, 74(1), 33-47. 
Kupsch, D., Waltert, M., \& Heymann, E. (2014). Forest type affects prey foraging of saddleback tamarins, Saguinus nigrifrons. Primates, 55(3), 403-413.

Lawson, C. R., Mann, D. J., \& Lewis, O. T. (2012). Dung beetles reduce clustering of tropical tree seedlings. Biotropica, 44(3), 271-275.

Leishman, M., Wright, I., Moles, A., \& Westoby, M. (2000). The evolutionary ecology of seed size. In M. Fenner (Ed.), Seeds: The ecology of regeneration in plant communities (pp. 31-57). Wallingford: CAB International.

Levey, D. J. (1987). Seed size and fruit-handling techniques of avian frugivores. The American Naturalist, 129(4), 471-485.

Lugon, A. P., Boutefeu, M., Bovy, E., Vaz-de-Mello, F. Z., Huynen, M.-C., Galetti, M., et al. (2017). Persistence of the effect of frugivore identity on post-dispersal seed fate: consequences for the assessment of functional redundancy. Biotropica, 49(3), 293-302.

McConkey, K. R., \& Brockelman, W. Y. (2011). Nonredundancy in the dispersal network of a generalist tropical forest tree. Ecology, 92(7), 1492-1502.

McNair, J. N., Sunkara, A., \& Frobish, D. (2012). How to analyse seed germination data using statistical timeto-event analysis: Non-parametric and semi-parametric methods. Seed Science Research, 22(2), 77-95.

Nathan, R., \& Muller-Landau, H. (2000). Spatial patterns of seed dispersal, their determinants and consequences for recruitment. Trends in Ecology \& Evolution, 15(7), 278-285.

Nickle, D. A., \& Heymann, E. W. (1996). Predation on Orthoptera and other orders of insects by tamarin monkeys, Saguinus mystax mystax and Saguinus fuscicollis nigrifrons (Primates: Callitrichidae), in northeastern Peru. Journal of Zoology, 239(4), 799-819.

Peres, C. A. (1993). Diet and feeding ecology of saddle-back (Saguinus fuscicollis) and moustached (S. mystax) tamarins in an Amazonian terra firme forest. Journal of Zoology, 230(4), 567-592.

Pons, T. L. (2000). Seed responses to light. In M. Fenner (Ed.), Seeds: The ecology of regeneration in plant communities (pp. 237-260). Wallingford: CAB International.

Razafindratsima, O. H., \& Dunham, A. E. (2014). Assessing the impacts of nonrandom seed dispersal by multiple frugivore partners on plant recruitment. Ecology, 96(1), 24-30.

Santos-Heredia, C., Andresen, E., \& Zárate, D. A. (2010). Secondary seed dispersal by dung beetles in a Colombian rain forest: Effects of dung type and defecation pattern on seed fate. Journal of Tropical Ecology, 26, 355-364.

Schupp, E. W. (1993). Quantity, quality and the effectiveness of seed dispersal by animals. Vegetatio, 107(108), 15-29.

Schupp, E. W., Jordano, P., \& Gómez, J. M. (2010). Seed dispersal effectiveness revisited: A conceptual review. New Phytologist, 188(2), 333-353.

Shepherd, V. E., \& Chapman, C. A. (1998). Dung beetles as secondary seed dispersers: Impact on seed predation and germination. Journal of Tropical Ecology, 14, 199-215.

Smith, A. C. (2000). Composition and proposed nutritional importance of exudates eaten by saddleback (Saguinus fuscicollis) and mustached (Saguinus mystax) tamarins. International Journal of Primatology, 21(1), 69-83.

Soini, P., \& Coppula, M. (1981). Ecología y dinámica poblacional de pichico Saguinus fuscicollis (Primates, Callitrichidae). Informe de Pacaya, 4, 1-43.

Soini, P., \& Soini, M. (1982). Distribución geográfica y ecología poblacional de Saguinus mystax (Primates, Callitrichidae). Informe de Pacaya, 6, 1-56.

Stevenson, P. R. (2011). Pulp-seed attachment is a dominant variable explaining legitimate seed dispersal: A case study on woolly monkeys. Oecologia, 166, 693-701.

Stiles, E. (2000). Animals as seed dispersers. In M. Fenner (Ed.), Seeds: The ecology of regeneration in plant communities (pp. 111-124). Wallingford, UK: CAB International.

Therneau, T. (2014). A Package for Survival Analysis in S. R package version, 2, 37-37 http://CRAN.Rproject.org/package=survival.

Traveset, A., Bermejo, T., \& Willson, M. (2001). Effect of manure composition on seedling emergence and growth of two common shrub species of Southeast Alaska. Plant Ecology, 155, 29-34.

Traveset, A., Robertson, A. W., \& Rodríguez-Pérez, J. (2007). A review on the role of endozoochory in seed germination. In A. J. Dennis, E. W. Schupp, R. J. Green, \& D. A. Westcott (Eds.), Seed dispersal: Theory and its application in a changing world (pp. 78-103). Wallingford: CAB International.

Vander Wall, S. B., \& Longland, W. S. (2004). Diplochory: Are two seed dispersers better than one? Trends in Ecology \& Evolution, 19(3), 155-161.

Vulinec, K. (2000). Dung beetles (Coleoptera: Scarabaeidae), monkeys, and conservation in Amazonia. Florida Entomologist, 83(3), 229-241.

Wang, B. C., \& Smith, T. B. (2002). Closing the seed dispersal loop. Trends in Ecology \& Evolution, 17(8), 379-385. 